\title{
3 Research Square

\section{Effects of an on-Table Reconstruction Technique Combined with Miniplate Internal Fixation and Prosthesis for the Treatment of Isolated Mason III Radial Head Fractures}

HAN Lei

Xiaoshan Traditional Chinese Medical Hospital

WANG Can-feng

Xiaoshan Traditional Chinese Medical Hospital

HU Yun-geng

Xiaoshan Traditional Chinese Medical Hospital

FAGN Wei-li

Xiaoshan Traditional Chinese Medical Hospital

Song Zhou-feng ( $\square$ songzhoufeng6505@163.com )

The First Affiliated Hospital of Zhejiang Chinese Medical University

\section{Research Article}

Keywords: radial head fracture, fracture fixation, prosthesis, Mason III

Posted Date: January 10th, 2022

DOI: https://doi.org/10.21203/rs.3.rs-488976/v2

License: (c) (1) This work is licensed under a Creative Commons Attribution 4.0 International License.

Read Full License 


\section{Abstract \\ Background}

In this retrospective study we analyzed a consecutive series of patients affected by isolated radial head Mason III fractures and treated with an on-table reconstruction technique combined with miniplate internal fixation or prosthesis.

\section{Methods}

This study included 42 patients affected by isolated radial head Mason III fractures and treated between Jan 2012 and Jan 2019. Twenty-four patients (average age 45.6 years) were treated with an on-table reconstruction technique combined with miniplate internal fixation. The remaining 18 (average age 44.5 years) were treated with prosthesis. From a clinical point of view, we evaluated the patients according to the Broberg-Morrey scoring system and the (disabilities of the arm, shoulder, and hand) DASH questionnaire for parameters.

\section{Results}

We found similar results in both the groups according to Broberg-Morrey score system and the DASH questionnaire at the last follow-up. The scores of the prosthesis group were better than those of the internal fixation group at the 1 st, 3rd, and 6 th month follow-up post-operation.

\section{Conclusion}

Both the on-table reconstruction technique combined with the miniplate internal fixation and prosthesis can be effective in treating patients with isolated Mason III radial head fractures. There was no difference in elbow function between the two groups at the last follow-up; however, a prosthesis favors early functional recovery of the elbow.

\section{Highlights}

- Capitellectomy can decrease the anti-valgus ability of elbow joints and cause other complications.

- The on-table reconstruction technique with mini-plate internal fixation and prosthesis can effectively treat isolated Mason III radial head fractures.

- Prosthesis offers early functional recovery of the elbow after isolated Mason III radial head fractures $\llbracket$

- A patient's age, elbow function requirements, and economic factors must be considered before concluding if a prosthesis is the best treatment. 


\section{Background}

A radial head fracture is a common injury of the elbow joint, accounting for more than $30 \%$ of all elbow fractures ${ }^{[1]}$. The treatment of radial head fractures is based on the fracture type and the presence of any associated injury.Masson classification is commonly used for radial head fractures. There is little question that a

Mason type I fracture, because of nondisplacement, should be managed without surgery. Open reduction and internal fixation has been a preferred treatment option for simple Mason II fractures and has given excellent results, but its use in the treatment of type III fractures remains controversial ${ }^{[2]}$. Highly comminuted complex Mason III fractures that cannot be reconstituted have poor outcomes when treated by open

reduction and internal fxation ${ }^{[3]}$. Capitellectomy can cause elbow varus deformity, radial displacement, unstable elbow joints, and other complications ${ }^{[4]}$. Therefore, prosthesis are often used in the treatment of Mason III fractures ${ }^{[5]}$. However,

complications described in prosthesis are in common in almost all the papers, aseptic mobilization, erosion of the capitellum,osteoarthritis, and heterotopic ossifcation ${ }^{[6]}$.

The management of this issue remains a matter of controversy. This retrospective study was to assess two of the treatments for isolated Mason III fractures. We analyzed the clinical results between 2012 and 2019 , obtained remotely, from on table reconstruction combined with miniplate fixation and prosthesis of radial head fractures. All patients obtained informed consent before participating in the clinical trial. This study was approved by the Ethics Committee of Xiaoshan District Hospital of TCM (No. 2012011).

\section{Patients And Methods}

All study participants provided informed consent. Forty-two patients surgically treated for radial head isolated Masson III fractures between Jan 2012 to Jan 2019 were selected for this study(Fig. 1). There were 27 males and 15 females with an average age of 45.2 years (range: 31-68 years). All the patients included in the study were affected by isolated Masson III radial head fractures without any other lesion affecting the interested elbow. The patients were divided into two groups, group $A$ and $B$ depending on the different treatment methods. The group A consisted of 24 patients were treated with miniplate internal fixation in combination with the on-table reconstruction technique. Among the remaining 18 patients in group B and they were treated with prosthesis.

\section{Perioperative preparation}

The affected side was immobilized before the operation, and the preoperative examinations, including elbow and forearm X-ray and elbow CT(Fig. 2a), to clarify the fracture classification and combined injury, were conducted. 


\section{Surgical methods}

All the operations were performed by the same surgeons. Group A and group B underwent surgery using the methodology discussed below.

Group A: the skin and subcutaneous tissue were incised using the Kocher approach. The forearm was kept pronated to avoid injury to the deep branch of the radial nerve. The radial head was fully exposed after incising the annular ligament. All the precise fracture pieces were anatomically reduced on the operating table(Fig. 2b). The larger fracture pieces were fixed with screws or Herbert nails(Fig. 2c), and the smaller ones were temporarily fixed with a 0.8-mm Kirschner wire. Extremely small fracture pieces were removed. A miniplate was used to fix and reconstruct the articular surface of the radial head. Fracture reduction was observed under direct vision and C-arm fluoroscopy in order to ensure that the screw or the Kirschner wire did not pass through the articular cartilage surface. The annular ligament, joint capsule, and lateral collateral ligament were repaired with a direct suture or anchor to strengthen the stability of the lateral elbow joint. For those with an obvious bone defect of the radial neck, the autogenous bone was taken from the lateral condyle of the humerus to prevent nonunion and displacement.

Group B: The surgical incision was the same as in group A. The broken bones were removed, and the radial head bone blocks were spliced on the prosthesis template. A suitable prosthesis model was selected according to its diameter. The bone marrow cavity of the proximal radius was repaired and polished with a special medullary cavity file to facilitate the implantation of the prosthesis. The proximal end of the radius was removed smoothly so that the radius and the neck of the prosthesis could be completely anastomosed. The medullary cavity was reamed with a cavity file from small to large. After the prosthesis test model was placed and the stability of the brachioradial joint was confirmed, the matching artificial radial head (Zimmer) was placed into the medullary cavity of the radial neck, and the elbow joint was passively flexed and extended. The elbow joint was checked for good activity and medial and lateral stability(Fig. 3a,3b,3c). C-arm fluoroscopy confirmed that the prosthesis position was satisfactory and the humeroradial joint position was good.

\section{Postoperative treatment}

Oral indomethacin, $25 \mathrm{mg} /$ day, was administered postoperatively to prevent heterotopic ossification. The elbow joints in group A could be actively and passively flexed and extended on the second day after the operation. The forearm rotation movement began 2 weeks after the operation; load-bearing activities were avoided for 4 weeks post-operation. According to the situation of fracture healing, weight-bearing activities began 8 weeks after the operation. The patients in group B gradually carried out active and passive activities of the elbow joints on the second day after the operation, and gradually increased muscle strength training around the elbow.

\section{Follow-up and evaluation of efficacy}


The fracture healing and prosthesis position were observed by X-ray. We evaluated the patients according to the Broberg-Morrey score system ${ }^{[7]}$ and the DASH questionnaire[8]. The Broberg-Morrey functional score was mainly based on the elbow joint and forearm flexion and extension, rotational range of motion, muscle strength, stability, and subjective pain. The range of motion was 40 points, strength was 20 points, stability was 5 points, and pain was 35 points, adding up to 100 points. The DASH questionnaire was used to evaluate the patients' upper limb functions, based on the degree of postoperative limb function limitation; 0 meant that the function was completely normal and 100 meant that there was no function

\section{Statistical analysis}

SPSS v. 22 was used for data analysis. For continuous quantitative data, approximately normal distributions are presented as mean and standard deviation (mean $\pm S D$ ) and the values were evaluated via an independent t-test. Median was used to describe skewed distributions and between-group differences were assessed via the independent Mann-Whitney $U$ test; the values were analyzed via Pearson chi-square or Fisher's exact test. Statistical significance was defined as $P<0.05$.

\section{Results}

\section{General conditions}

Epidemiological showed that the mean age, gender, operative time, hospital stay, time to operation, and the follow-up period in both groups are comparable $(p>0.05)$. Details of the epidemiology and perioperative parameters are listed in Table 1. No radial head necrosis was found in the two groups and one case of traumatic arthritis was found in group A. No infection, nerve injury, internal fixation, or prosthesis loosening occurred in both groups.

Table 1. Epidemiology and perioperative parameters of radial head fractures

\begin{tabular}{llll}
\hline & Group A & Group B & P \\
\hline Number of patients & 24 & 18 & \\
\hline Mean age, years & $45.6(31-68)$ & $44.5(35-66)$ & 0.135 \\
\hline Gender & & & \\
$\quad$ Male & 15 & 12 & 0.527 \\
$\quad$ Female & 9 & 6 & \\
\hline $\begin{array}{l}\text { Fracture laterality } \\
\quad \text { Left side }\end{array}$ & 11 & 7 & 0.632 \\
$\quad$ Right side & 13 & 11 & \\
\hline Time to operation, days (range) & $4.5(2-7)$ & $4.1(2-8)$ & 0.232 \\
\hline Operative time, minutes (range) & $60(40-95)$ & $55(45-80)$ & 0.372 \\
\hline Hospital stay, days (range) & $8.2(6-14)$ & $8.5(5-14)$ & 0.118 \\
\hline Follow-up, months (range) & $12.5(8-35)$ & $14.2(6-36)$ & 0.197 \\
\hline
\end{tabular}


P-value $<0.05$. No statistically significant difference between two groups in terms of epidemiology and perioperative parameters

\section{Clinical efficacy evaluation}

According to the Broberg-Morrey scoring system, the scores of group A were $80.6 \pm 6.2,81.9 \pm 5.8,84.4 \pm 5.2$ and $91.2 \pm 8.1$ at the end of the $1 \mathrm{st}, 3 \mathrm{rd}, 6$ th month and the last follow up post-operation, respectively. Meanwhile, the scores of group B were 84.5 $\pm 7.2,86.8 \pm 6.4,89.6 \pm 3.8$ and $92.1 \pm 7.4$. The DASH scores of group A were $25.1 \pm 4.7,21.4 \pm 4.2,19.2 \pm 3.7$ and $11.5 \pm 3.1$ at the end of the $1 \mathrm{st}, 3 \mathrm{rd}$, 6 th month and at the last follow up post-operation. Meanwhile, the scores of group B were 21.1 $\pm 5.2,17.5 \pm 4.1,13.3 \pm 3.1$, and 12.1 \pm 3.4 . The difference of the Broberg-Morrey and DASH scores between these two groups was statistically significant at the $1 \mathrm{st}$, 3rd, and 6 th month follow-up $(p<0.05)$, but not statistically significant $(p>0.05)$ at the last follow-up.

\section{Discussion}

Mason type III fracture of the radial head is defifined as a comminuted, displaced fracture involving the entire radial head ${ }^{[9]}$.For Masson III radial head fractures, capitellectomy is often used in the early stages. Although a satisfactory range of motion of the elbow joint can be obtained in the early stage, the stability of the elbow joint is significantly decreased in the long-term ${ }^{[10]}$. With increased knowledge of the applied anatomy and biomechanics of the elbow joint, the radial head is considered an important structure to maintain lateral stability of the elbow joint and transmit load ${ }^{[11]}$. Excision can cause elbow varus deformity, radial displacement, unstable elbow joint and other complications ${ }^{[4]}$. Due to the radial head's contribution to elbow stability, in absence of other bony or ligamentous lesions both internal fixation and prosthesis can be good treatment in these kinds of fractures ${ }^{[12,13]}$.

However, a prosthesis is expensive, and prosthesis loosening, sinking, and prosthesis wear may occur in the long-term after surgery ${ }^{[6]}$. It is necessary to consider the patient's age, elbow function requirements, and economic factors comprehensively before concluding if a prosthesis is the best treatment. When considering a prosthesis, the following points should be addressed: 1) the osteotomy plane must be accurately grasped. If it is too high, the implanted prosthesis will be tight. If it is too low, the implanted prosthesis will be unable to make contact with the capitellum and result in an unsatisfactory surgical effect; 2) selecting the appropriate radial head prosthesis. If the diameter of the radial head prosthesis does not match that of the upper radioulnar joint, it can lead to the cam effect and affect the rotation center of the elbow joint; 3 ) it is necessary to repair the anterior joint capsule, lateral collateral ligament, and extensor insertion. Checking the stability of the elbow joint with the varus and valgus tension during the operation, to ensure the dynamic and static stability of the elbow joint to the greatest extent, is very important. 
The on-table reconstruction technique refers to the removal of all the fracture pieces of the radial head and precise reduction under direct vision on the operating table. As Masson III fractures involving the articular surface of the radial head are often more than three pieces, the in vivo space for reduction is narrow, and reduction is usually more difficult ${ }^{[14]}$. In our study, the larger fracture fragments were removed out and reduced by screws, the small fracture fragments were temporarily fixed with multiple fine Kirschner wires. The radial head was then put back into the elbow joint cavity for miniplate fixation after complete external splicing. The literature reports that this technique does not increase the probability of ischemic necrosis of the radial head ${ }^{[14,15]}$. In the past, it was considered that the reduction and fixation were difficult in patients with more than 3 pieces of fracture and the failure rate of internal fixation and the probability of nonunion were high. However, in recent years, with the clinical application of the ontable reconstruction technology for Masson III radial head fractures, open reduction and miniplate fixation can obtain satisfactory clinical efficacy ${ }^{[14,15]}$. The miniplate with a low notch has good flexibility and elasticity. It is easy to shape during operation and can provide good stability ${ }^{[16]}$. However, the disadvantages of on-table reconstruction combined with miniplate fixation are as follows: 1) patients with obvious bone defects are more likely to form fracture non-union at a later stage; 2) for multiple elbow injuries, it generally needs to be fixed for 2-4 weeks, and too long a braking time is not conducive to the early functional exercise of the elbow.

There are several limitations in this study, including its retrospective nature, the small sample size, and the short follow-up time. The medium and long-term efficacy and safety need to be further confirmed in future studies. A randomized prospective trial with a large sample size and long-term follow-up is required to make more definitive conclusions.

\section{Conclusion}

According to this study and the most recent literature, both the on-table reconstruction technique combined with mini-plate internal fixation and a prosthesis can be effective in treating patients with isolated Mason III radial head fractures. There was no difference in the elbow function between the two groups at the last follow-up, but a prosthesis offers early functional recovery of the elbow $\square$

\section{Declarations}

\section{Acknowledgments}

The authors would like to thank all participating patients, as well as the study nurses, co-investigators, and colleagues who made this trial possible.

\section{Authors' contributions}

$\mathrm{HL}$ was accountable for the integrity and analysis of the data and the writing of the manuscript. WCF, HYG and FWL were accountable for the integrity and analysis of the data. SZF was accountable for the 
conception of the research. All authors read and approved the final manuscript.

\section{Funding}

There is no funding source

\section{Ethics approval and consent to participate}

This study was conducted in accordance with the Declaration of Helsinki.

This study was conducted with approval from the Ethics Committee of Xiaoshan District Hospital of TCM and The First Affiliated Hospital of Zhejiang Chinese Medical University (reference number 2012011). Written informed consent was obtained from all participants.

\section{Consent for publication}

Written informed consent was obtained from all participants.

\section{Competing interests}

The authors declare that they have no competing interests. There is no funding source.

\section{References}

1. van Riet RP, van den Bekerom M, Van Tongel A, Spross C, Barco R, Watts AC.Radial head fractures.Shoulder Elbow,2020 Jun;12(3):212-223. doi: 10.1177/1758573219876921.

2. J Pegrum,S Isaac. Open Reduction and Internal Fixation Versus Radial Head Arthroplasty for Mason III Radial Head Fractures: Appraising the Current Literature Evidence.Cureus.2020 Apr 1;12(4):e7501. doi: 10.7759/cureus.7501.

3. Hong Jiang Ruan,Cun Yi Fan,Jun Jian Liu, Bing fang Zeng.A comparative study of internal fixation and prosthesis replacement for radial head fractures of Mason type III International Orthopaedics (SICOT) (2009) 33:249-253 doi: 10.1007/s00264-007-0453-3.

4. Bonnevialle N. Radial head replacement in adults with recent fractures. OTSR. 2016;102(1):S69-S79. doi: 10.1016/j.otsr.2015.06.026.

5. Chen HW, Tian JL, Zhang YZ.Therapeutic Effect of Resection, Prosthetic Replacement and Open Reduction and Internal Fixation for the Treatment of Mason Type III Radial Head Fracture.J Invest Surg. 2021 Jan;34(1):30-38. doi: 10.1080/08941939.2019.1602689.

6. Seung Min Ryu, Sam-Guk Park, Ji-Hoon Kim,Han Seok Yang, Ho Dong Na, Jae-Sung Seo.Treatment of Modified Mason Type III or IV Radial Head Fracture: Open Reduction and Internal Fixation versus Arthroplasty.Indian J Orthop. 2018 Nov-Dec; 52(6): 590-595. doi: 10.4103/ortho.IJOrtho_537_16

7. Broberg MA,Money BF.Results of treatment of fracture dislocations of the elbow[J].Clin Orthop Relat Res,1987,(216):109-119ه doi: 10.1097/00003086-198703000-00017ه 
8. Gummesson C,Atroshi I,Ekdahl C.The disabilities of the arm,shoulder and hand(DASH) out come questionnaire冈longitudinal construct validity and measuring self-rated health change after surgery.BMC Musculoskelet Disord,2003,4:11. doi: 10.1186/1471-2474-4-11.

9. Ring D, Quintero J, Jupiter J. Open reduction and internal fifixation of fractures of the radial head. J Bone J Surg Am. 2002;84A:1811-1815. doi: 10.2106/00004623-200210000-00011

10. N. Bonnevialle, Radial head replacement in adults with recent fractures. Orthop Traumatol Surg Res,2016 Feb;102(1 Suppl):S69-79. doi:10.1016/j.otsr.2015.06.026.

11. Swensen SJ, Tyagi V, Uquillas C, Shakked RJ, Yoon RS, Liporace FA.Maximizing outcomes in the treatment of radial head fractures.J Orthop Traumatol. 2019 Mar 23;20(1):15. doi: 10.1186/s10195019-0523-5.

12. R Grewal,JC Macdermid,KJ Faber,DS Drosdowech,GJW King. Comminuted radial head fractures treated with a modular metallic radial head arthroplasty.J Bone Joint Surg Am.2006 Oct;88(10):2192200. doi: 10.2106/JBJS.E.00962.

13. Hongwei Chen, Ziyang Wang, Yongjun Shang.Clinical and Radiographic Outcomes of Unipolar and Bipolar Radial Head Prosthesis in Patients with Radial Head Fracture: A Systemic Review and MetaAnalysis.Invest Surg. 2018 Jun;31(3):178-184. doi: 10.1080/08941939.2017.1299262.

14. G.N.Kiran Kumar, Gaurav Sharma, Kamran Farooque, Vijay Sharma, Vaibhav Jain, Ravijot Singh.Ontable reconstruction and fixation of Mason type III radial head fractures.Chin J Traumatol. 2015;18(5):288-92. doi: 10.1016/j.cjtee.2015.11.005.

15. Businger A, Ruedi TP, Sommer C. On-table reconstruction of comminuted fractures of the radial head. Injury. 2010;41:583-588. doi: 10.1016/j.injury.2009.10.026.

16. Ikeda $M$, Yamashina $Y$, Kamimoto $M, O$ Oka Y.Open reduction and internal fixation of comminuted fractures of the radial head using low-profile mini-plates.J Bone Joint Surg Br.2003 Sep;85(7):1040-4. doi: 10.1302/0301-620x.85b7.13823.

\section{Figures}




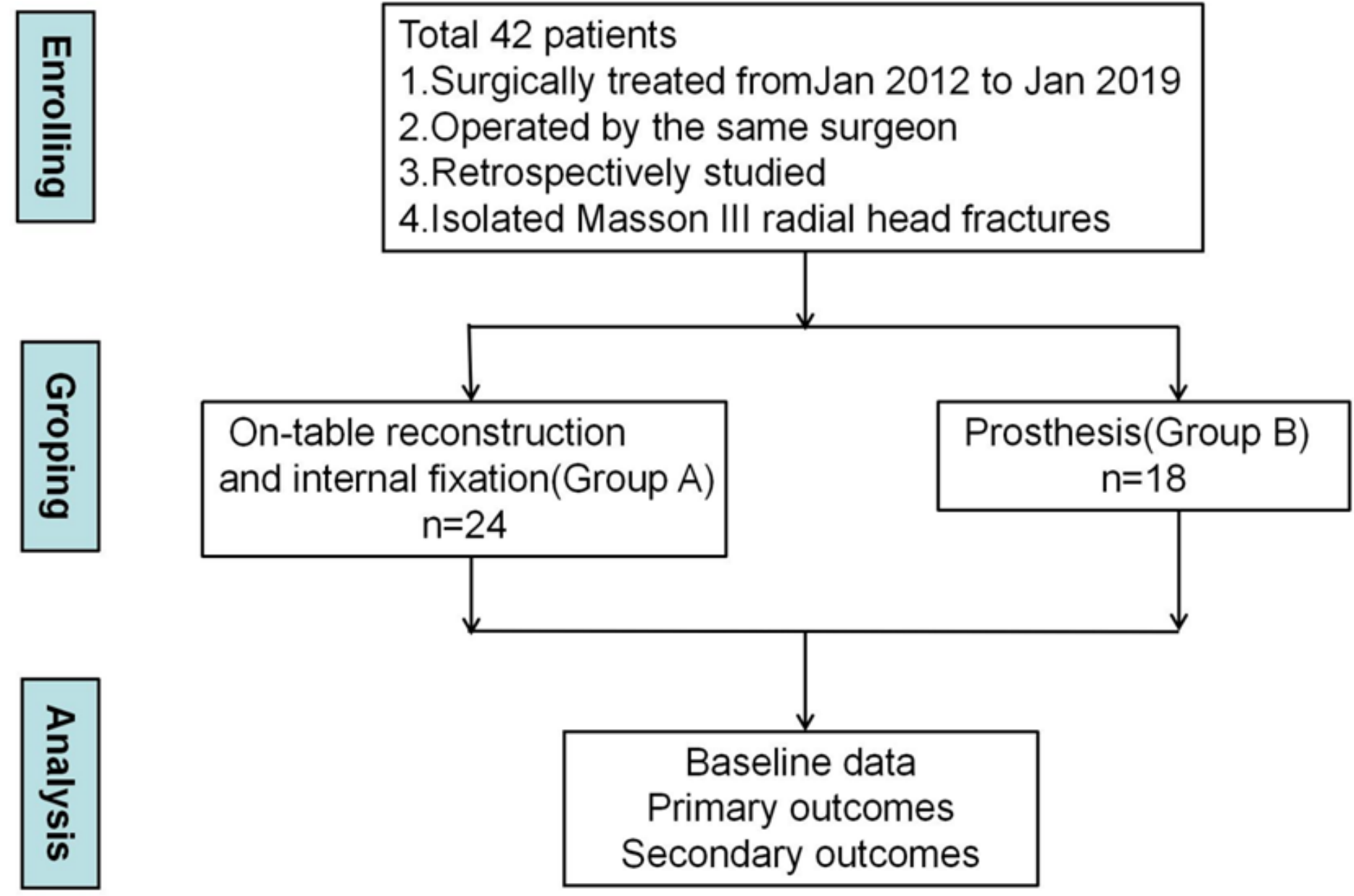

Figure 1

A flow chart showing number of patients in this study. 

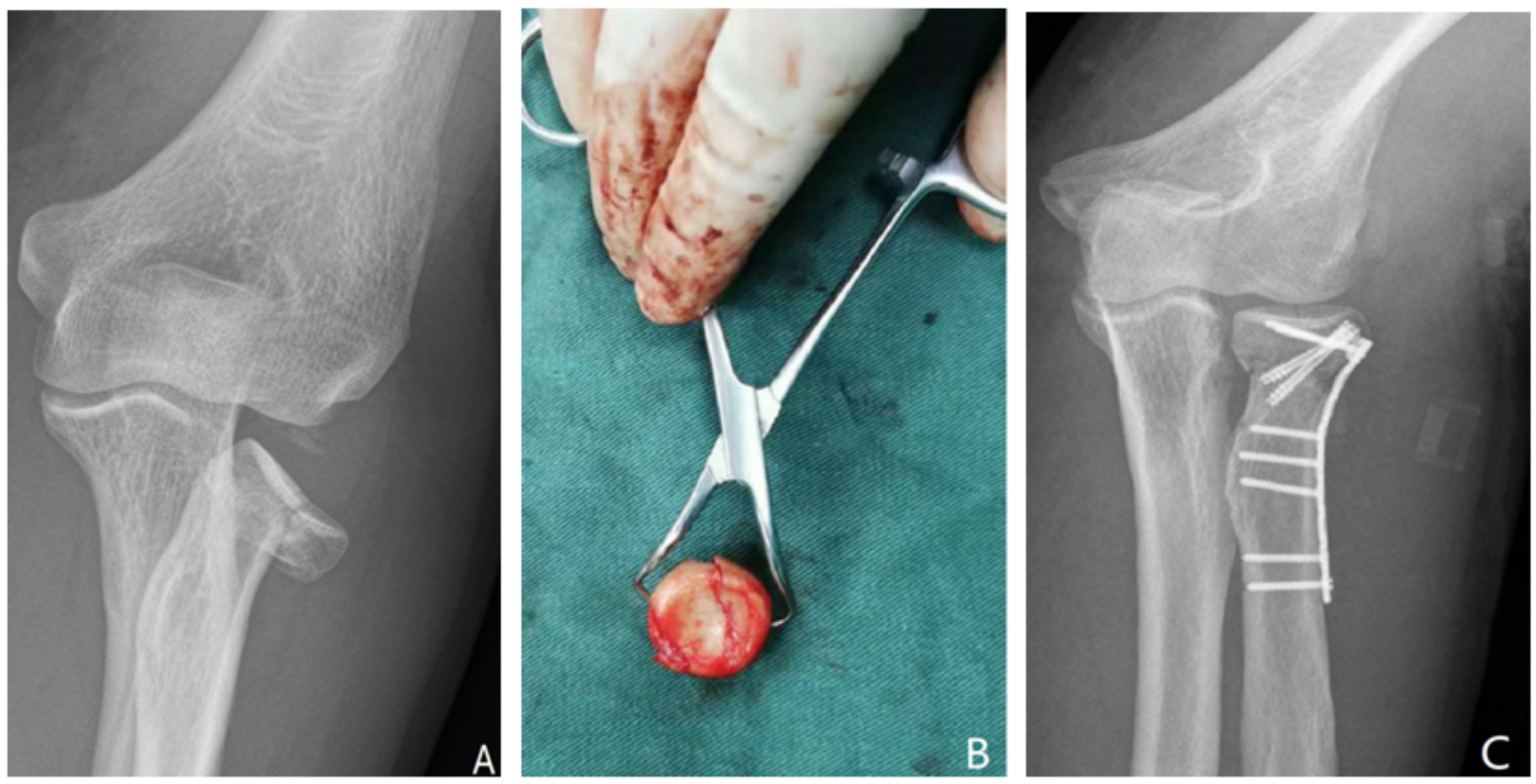

Figure 2

Preoperative X-ray showing Mason III radial head fracture (A), on-table reconstruction with preliminary fixation (B), postoperative $\mathrm{X}$-ray showed that the fracture was reduced and the internal fixation position was satisfactory $(\mathrm{C})$.
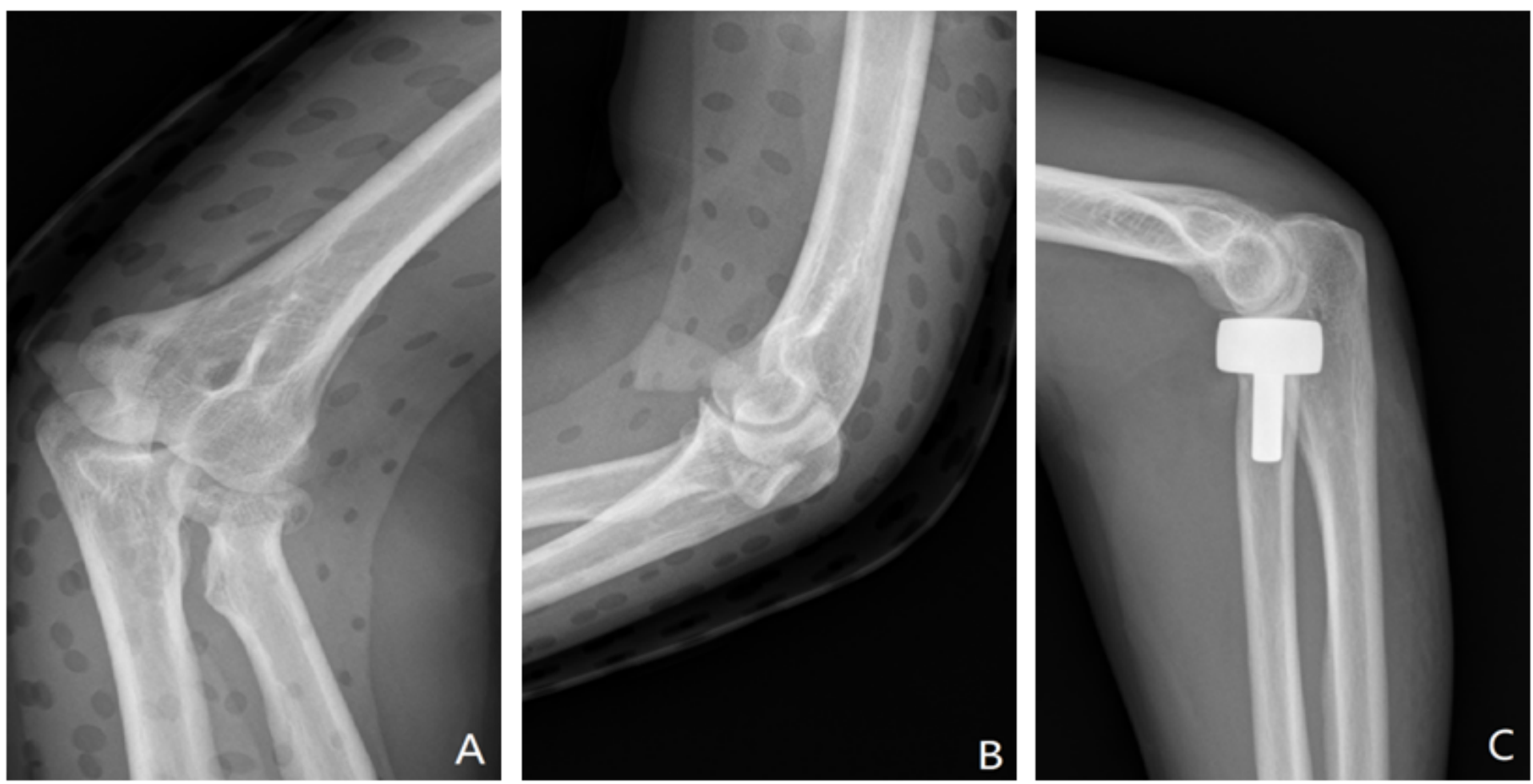

Figure 3 
Anteroposterior (A) and lateral (B) X-ray images of a patient with Mason III radial head fracture. Replacement by radial head prosthesis(C). 